\title{
Factors Associated with a Prolonged Length of Hospital Stay in Patients with Diabetic Foot: A Single-Center Retrospective Study
}

\author{
Sang Kyu Choi ${ }^{1}$, Cheol Keun Kim ${ }^{1}$, Dong In Jo ${ }^{1}$, Myung Chul Lee ${ }^{2}$, Jee Nam Kim², \\ Hyun Gon $\mathrm{Choi}^{2}$, Dong Hyeok Shin ${ }^{2}$, Soon Heum Kim ${ }^{1}$ \\ ${ }^{1}$ Department of Plastic and Reconstructive Surgery, Konkuk University Chungju Hospital, Chungju; ${ }^{2}$ Department of Plastic and \\ Reconstructive Surgery, Konkuk University Seoul Hospital, Konkuk University School of Medicine, Seoul, Korea
}

Background We conducted this study to identify factors that may prolong the length of the hospital stay (LHS) in patients with diabetic foot (DF) in a single-institution setting.

Methods In this single-center retrospective study, we evaluated a total of 164 patients with $\mathrm{DF}$, and conducted an intergroup comparison of their baseline demographic and clinical characteristics, including sex, age, duration of diabetes, smoking status, body mass index, underlying comorbidities (e.g., hypertension or diabetic nephropathy), wound characteristics, type of surgery, the total medical cost, white blood cell (WBC) count, C-reactive protein (CRP) levels, erythrocyte sedimentation rate, and albumin, protein, glycated hemoglobin, and 7-day mean blood glucose (BG) levels.

Results Pearson correlation analysis showed that an LHS of $>5$ weeks had a significant positive correlation with the severity of the wound $(r=0.647)$, WBC count $(r=0.571)$, CRP levels $(r=0.390)$, DN $(r=0.020)$, and 7-day mean BG levels $(r=0.120)(P<0.05)$. In multiple regression analysis, an LHS of $>5$ weeks had a significant positive correlation with the severity of the wound (odds ratio $[\mathrm{OR}]=3.297 ; 95 \%$ confidence interval $[\mathrm{Cl}], 1.324-10.483$; $\mathrm{P}=0.020)$, WBC count $(\mathrm{OR}=1.423 ; 95 \% \mathrm{Cl}, 0.046-0.356 ; \mathrm{P}=0.000)$, $C R P$ levels $(\mathrm{OR}=1.079$; $95 \% \mathrm{Cl}, 1.015-1.147 ; \mathrm{P}=0.014)$, albumin levels $(\mathrm{OR}=0.263 ; 95 \% \mathrm{Cl}, 0.113-3.673 ; \mathrm{P}=0.007)$, and 7-day mean $B G$ levels $(O R=1.018 ; 95 \% \mathrm{Cl}, 1.001-1.035 ; \mathrm{P}=0.020)$.

Conclusions Surgeons should consider the factors associated with a prolonged LHS in the early management of patients with DF. Moreover, this should also be accompanied by a multidisciplinary approach to reducing the LHS.

Keywords Diabetes mellitus / Diabetic foot / Length of stay / Prognosis / Limb salvage
Correspondence: Soon Heum Kim Department of Plastic and Reconstructive Surgery, Konkuk University Chungju Hospital, Konkuk University School of Medicine, 82 Gugwon-daero, Chungju 27376, Korea

Tel: +82-43-840-8860

Fax: +82-43-840-9862

E-mail: plastic7@kku.ac.kr

Received: 18 Jul 2017 • Revised: 11 Oct 2017 • Accepted: 31 Oct 2017

pISSN: 2234-6163 • elSSN: 2234-6171 • https://doi.org/10.5999/aps.2017.01207• Arch Plast Surg 2017;44:539-544

\section{INTRODUCTION}

According to a 2012 report, the prevalence of diabetes mellitus (DM) will gradually rise and the number of patients with DM will exceed 5.9 million by the year 2030 [1]. It has been reported that the annual incidence of diabetic foot (DF), resulting in foot ulcer and gangrene, is estimated to be approximately $2 \%-$ $5 \%$ in the general population, and its lifetime risk is known to be

Copyright $(\odot) 2017$ The Korean Society of Plastic and Reconstructive Surgeons

This is an Open Access article distributed under the terms of the Creative Commons Attribution Non-Commercial License (http://creativecommons.org/

licenses/by-nc/4.0/) which permits unrestricted non-commercial use, distribution, and reproduction in any medium, provided the original work is properly cited.

www.e-aps.org 
$15 \%-20 \%$. Moreover, $15 \%$ of patients with DF may undergo lower extremity amputation (LEA) and about $20 \%$ of the total medical expenses for diabetes are paid for the treatment of DF. Furthermore, the mortality of patients with DF is twice as high as other individuals with DM and they are at a 10-15 times higher risk of undergoing LEA. It has also been reported that patients with DF account for $40 \%-70 \%$ of total cases of nontraumatic LEA [2].

In patients with $\mathrm{DF}$, the treatment protocol requires the following factors: (1) good communication between patients and a multidisciplinary group of physicians and surgeons; (2) early management of patients based on evidence-based treatment recommendations; (3) weekly imaging studies of the wound accompanied by the relevant documentation; (4) objective assessment of blood flow in the lower extremity; (5) debridement of hyperkeratotic, infected, and nonviable tissue; (6) systemic antibiotic treatment as well as drainage for deep infection and cellulitis; (7) off-loading; (8) maintenance of a moist wound bed; (9) the use of growth factor and vacuum-assisted therapy for complex wounds [3].

Patients with DF ulcer accompanied by infections are commonly encountered in clinical settings. According to a literature review, the length of hospital stay (LHS) serves as an important outcome of DF $[4,5]$. Still, however, few studies have investigated LHS. Identifying factors associated with treatment outcomes would make it possible to better predict a patient's prognosis and to reduce the socioeconomic burden of DF.

With the above considerations in mind, we conducted this study to identify factors that may prolong the LHS in patients with DF in a single-institution setting.

\section{METHODS}

\section{Study patients and setting}

The current single-center retrospective study was conducted among 164 patients with DF $(\mathrm{n}=164)$ who underwent surgery at Konkuk University Chungju Hospital during the 6-year period between March 2011 and February 2016.

The inclusion criteria for the current study were as follows: (1) Men or women aged 18 years or older; (2) Patients with a confirmed diagnosis of type I or II DM according to the guidelines of the American Diabetes Association; (3) Patients receiving pharmacological treatment for glycemic control; (4) Patients with an infected wound requiring hospitalization and a history of receiving surgical debridement at least twice; (5) Patients who were transferred to an intensive care unit or referred to other departments for further evaluation and treatment of DF sepsis.

The exclusion criteria for the current study were as follows: (1)
Patients with comorbidities that may affect the course of DF (e.g., tumors or immunological disorders); (2) Women who were pregnant or breastfeeding; (3) Patients with an acute traumatic wound with an onset period of < 1 week; (4) Patients who were transferred to an intensive care unit or referred to other departments for further evaluation and treatment of conditions other than DF sepsis (e.g., hospital-acquired pneumonia); (5) Patients who refused to undergo surgery; (6) Patients who left the hospital against medical advice; (7) Patients who were treated later because of a treatment delay; (8) Patients who refused medical advice; (9) Patients who were readmitted within 2 weeks of discharge; (10) Patients who were deemed to be ineligible for study participation according to our judgment.

The current study was approved by the Institutional Review Board of Konkuk University Chungju Hospital medical institution (IRB no. 2014-042). All patients provided written informed consent.

\section{Treatment protocol}

On admission, patients with systemic signs and symptoms that were suggestive of an infected wound, such as redness, heat, induration, swelling, and purulent discharge, received clinical and laboratory examinations. In addition, measurements were made of the white blood cell (WBC) count, C-reactive protein (CRP) levels, erythrocyte sedimentation rate (ESR), and albumin, protein, glycated hemoglobin (HbAlc), and blood glucose (BG) levels.

After admission, the patients received wound preparations, which included wound irrigation, serial debridement, negativepressure wound therapy, and suturing for the purpose of reducing the dead and raw spaces.

\section{Patient evaluation and criteria}

For the current analysis, we defined the LHS as the duration between admission and the time of discharge, when the patients required no more surgical treatment according to the judgment of the surgeon or based on the presence of a small raw surface, and received conservative treatment, such as simple dressing and protection with plaster or a splint.

We performed a literature review to determine the optimal cut-off value for dividing our clinical series of patients, and found that the episode of care for DF was 5 weeks based on claims data [6]. We therefore divided the patients into 2 groups: one with an LHS $\leq 5$ weeks and another with an LHS $>5$ weeks. We compared the baseline and clinical characteristics between the 2 groups, attempting to identify factors that may have prolonged the LHS.

The baseline demographic and clinical characteristics of the 
patients included sex, age, duration of diabetes (the time elapsed between the initial diagnosis of diabetes and admission due to DF), smoking status (smoker or non-smoker), body mass index (BMI), underlying comorbidities (hypertension and diabetic nephropathy $[\mathrm{DN}]$ ), wound characteristics (location [weightbearing or non-weight-bearing], number of wounds $[1,2,3$, or $>3$ ], severity [involvement of skin, tendon, or muscle and bone], and duration), type of surgery (amputation, skin graft, or flap surgery), WBC count, CRP levels, ESR, and albumin, protein, $\mathrm{HbAlc}$, and 7-day mean BG levels.

\section{Statistical analysis}

All data are expressed as mean \pm standard deviation. Statistical analysis was done using SPSS ver. 23 (IBM Corp., Armonk, NY, USA). To identify factors that may have affected the LHS in each group, we performed a univariate analysis using Pearson correlation analysis. Moreover, we performed a multivariate analysis, in which factors found to be significant in the univariate analysis were included in the multiple regression analysis to identify factors that may have prolonged the LHS. For such factors, we calculated the odds ratio (OR) and $95 \%$ confidence interval (CI). A P-value of $<0.05$ was considered to indicate statistical significance.

\section{RESULTS}

\section{Baseline and clinical characteristics of the patients}

A total of 164 patients were enrolled in the current study; they consisted of 100 men and 64 women, and their mean age was $62.9 \pm 12.7$ years (range, $26-89$ years). Seventy-six patients had an LHS $\leq 5$ weeks, while 88 had an LHS $>5$ weeks. The baseline characteristics of the patients are presented in Table 1.

\section{Factors affecting the LHS in the Pearson correlation analysis}

In the Pearson correlation analysis, an LHS of $\leq 5$ weeks had a significant, strong, positive correlation with the severity of the wound $(\mathrm{r}=0.730)$ and a significant but moderate association with the WBC count $(r=0.472)$ and CRP levels $(r=0.443)$ $(\mathrm{P}<0.05)$. In addition, an LHS of $>5$ weeks had a significant, strong, positive correlation with the severity of the wound $(\mathrm{r}=0.647)$, a significant but moderate correlation with the WBC count $(\mathrm{r}=0.571)$, significant but weak correlations with CRP levels $(r=0.390)$ and DN $(r=0.020)$, and a significant but very weak correlation with 7-day mean BG levels $(\mathrm{r}=0.120)$ $(\mathrm{P}<0.05)$. In contrast, an LHS of $>5$ weeks had a significant, moderate, negative correlation with albumin levels $(\mathrm{r}=-0.420)$ $(\mathrm{P}<0.05)$ (Table 2).

\section{Table 1. Baseline and clinical characteristics of the patients}

\begin{tabular}{|c|c|}
\hline Characteristic & Values \\
\hline Age (yr) & $62.9 \pm 12.7$ \\
\hline \multicolumn{2}{|l|}{ Sex } \\
\hline Men & $100(60.9)$ \\
\hline Women & $64(39.1)$ \\
\hline Duration of DM (mo) & $240.5(1-480)$ \\
\hline LHS (day) & $31.0 \pm 32.0$ \\
\hline LHS $\leq 5$ wk & $76(46.3)$ \\
\hline LHS $>5$ wk & $88(52.7)$ \\
\hline Body mass index $\left(\mathrm{kg} / \mathrm{m}^{2}\right)$ & $25.8 \pm 4.2$ \\
\hline \multicolumn{2}{|l|}{ Smoking status } \\
\hline Smoking & $128(78.1)$ \\
\hline Non-smoking & $36(12.9)$ \\
\hline \multicolumn{2}{|l|}{ Underlying co-morbidities } \\
\hline HTN & $76(46.3)$ \\
\hline DN & 20 (12.2) \\
\hline \multicolumn{2}{|l|}{ Type of surgery } \\
\hline Amputation & $28(17.1)$ \\
\hline Skin graft & $72(43.9)$ \\
\hline Flap surgery & $64(39.0)$ \\
\hline \multicolumn{2}{|l|}{ Wound characteristics } \\
\hline \multicolumn{2}{|l|}{ Location } \\
\hline Weight-bearing areas & $126(75.9)$ \\
\hline Non-weight-bearing areas & $38(24.1)$ \\
\hline \multicolumn{2}{|l|}{ Number } \\
\hline 1 & $126(76.8)$ \\
\hline 2 & $20(12.2)$ \\
\hline 3 & $14(8.5)$ \\
\hline$>3$ & $4(2.4)$ \\
\hline \multicolumn{2}{|l|}{ Severity } \\
\hline Skin involvement & $62(37.8)$ \\
\hline Muscle or tendon involvement & $62(37.8)$ \\
\hline Bone involvement & $40(24.4)$ \\
\hline Duration (day) & $151.5(4-300)$ \\
\hline \multicolumn{2}{|l|}{ Laboratory measurements } \\
\hline WBC $\left(10^{3} / \mu \mathrm{L}\right)$ & $9.6 \pm 4.7$ \\
\hline $\mathrm{CRP}(\mathrm{mg} / \mathrm{dL})$ & $4.9 \pm 7.8$ \\
\hline $\mathrm{ESR}(\mathrm{mm} / \mathrm{hr})$ & $37.3 \pm 19.6$ \\
\hline Albumin (g/dL) & $3.5 \pm 0.8$ \\
\hline Protein (g/dL) & $6.4 \pm 1.6$ \\
\hline HbA1c (\%) & $9.22 \pm 5.7$ \\
\hline $\mathrm{BG}(\mathrm{mg} / \mathrm{dL})$ & $218.0 \pm 86.3$ \\
\hline \multicolumn{2}{|c|}{$\begin{array}{l}\text { Values are presented as mean } \pm \text { standard deviation, number (\%) or median and } \\
\text { range. } \\
\text { DM, diabetes mellitus; LHS, length of hospital stay; HTN, hypertension; DN, } \\
\text { diabetic nephropathy; WBC, white blood cell; CRP, C-reactive protein; ESR, } \\
\text { erythrocyte sedimentation rate; HDA1c, glycated hemoglobin; BG, blood glucose. }\end{array}$} \\
\hline
\end{tabular}

\section{Factors affecting the LHS in the multiple regression analysis}

In the multiple regression analysis, an LHS of $>5$ weeks had a significant positive correlation with the severity of the wound ( $\mathrm{OR}=3.297$; 95\% CI, 1.324-10.483; $\mathrm{P}=0.020)$, WBC count $(\mathrm{OR}=1.423$; 95\% CI, 0.046-0.356; $\mathrm{P}=0.000)$, CRP levels ( $\mathrm{OR}=1.079 ; 95 \% \mathrm{CI}, 1.015-1.147 ; \mathrm{P}=0.014)$, albumin levels $(\mathrm{OR}=0.263 ; 95 \% \mathrm{CI}, 0.113-3.673 ; \mathrm{P}=0.007)$, and 7-day mean 
Table 2. Factors affecting the length of hospital stay in the Pearson correlation analysis

\begin{tabular}{|lcc|}
\hline Characteristic & $\begin{array}{c}\text { LHS } \leq 5 \text { wk } \\
(\mathbf{n}=76)\end{array}$ & $\begin{array}{c}\text { LHS }>5 \text { wk } \\
(\mathbf{n}=\mathbf{8 8})\end{array}$ \\
\hline Baseline and clinical characteristics & & \\
Male sex & 0.082 & 0.106 \\
Age & 0.328 & 0.053 \\
Duration of DM & -0.113 & -0.195 \\
Smoking & -0.102 & -0.038 \\
BMl & 0.126 & 0.232 \\
HTN & -0.232 & -0.199 \\
DN & 0.014 & $0.020^{*}$ \\
Wound characteristics & & \\
Location & 0.098 & 0.200 \\
Number & 0.059 & 0.165 \\
Severity & $0.730^{*}$ & $0.647^{*}$ \\
Duration & 0.190 & 0.261 \\
Laboratory measurements & \\
WBC & $0.472^{*}$ & $0.571^{*}$ \\
CRP & $0.443^{*}$ & $0.390^{*}$ \\
ESR & 0.088 & 0.202 \\
Albumin & -0.073 & $-0.420^{*}$ \\
Protein & -0.122 & -0.027 \\
HbA1C & 0.197 & 0.021 \\
BG & 0.026 & $0.120^{*}$ \\
\hline Values are Pearson correlation coefficients. & \\
LHS, length of hospital stay; DM, diabetes mellitus; BMl, body mass index; HTN, \\
hypertension; DN, diabetic nephropathy; WBC, white blood cell; CRP, C-reactive \\
protein; ESR, erythrocyte sedimentation rate; HbA1c, glycated hemoglobin; BG, \\
blood glucose. \\
*Statistical significance at P<0.05 by Pearson correlation analysis. \\
\hline
\end{tabular}

BG levels (OR=1.018; 95\% CI, 1.001-1.035; P = 0.020) (Table 3).

\section{DISCUSSION}

Most of the patients enrolled in the current study were using an insulin pump due to difficulties with blood sugar control, and then received medical treatment for a poor general condition after admission. They had a prolonged LHS, with an average of $31.0 \pm 32.0$ days; this value is greater than has been reported in other published studies of similar case series. According to Wukich et al. [7], patients with DF accompanied by a severe infection had a mean LHS of 8 days. If accompanied by osteomyelitis, patients with DF should receive a long-term course of antibiotics.

In the current study, the severity of the wound had the strongest significant correlation with an LHS of $>5$ weeks. In patients with DF accompanied by osteomyelitis, surgeons should consider debridement before using antibiotics, which may prolong the treatment period in comparison with other types of wounds [8]. In other words, a minimum period of 2-4 weeks is required until the efficacy of antibiotics appears in patients with DF accompanied by osteomyelitis [9]. A maximum period of 3
Table 3. Factors affecting the length of hospital stay in the multiple regression analysis

\begin{tabular}{|lccc|}
\hline Characteristic & $\begin{array}{c}\text { Odds } \\
\text { ratio }\end{array}$ & $\begin{array}{c}\text { 95\% } \\
\text { Confidence } \\
\text { interval }\end{array}$ & P-value \\
\hline $\begin{array}{l}\text { Baseline and clinical characteristics } \\
\text { Diabetic nephropathy }\end{array}$ & 0.704 & $0.181-2.740$ & 0.613 \\
$\begin{array}{l}\text { Wound characteristics } \\
\quad \text { Severity }\end{array}$ & 3.297 & $1.324-10.483$ & $0.020^{*}$ \\
$\begin{array}{l}\text { Laboratory measurements } \\
\quad \text { White blood cell }\end{array}$ & 1.423 & $0.046-0.356$ & $0.000^{*}$ \\
$\quad$ C-reactive protein & 1.079 & $1.015-1.147$ & $0.014^{*}$ \\
$\quad \begin{array}{l}\text { Albumin } \\
\text { Blood glucose }\end{array}$ & 0.263 & $0.113-3.673$ & $0.007^{*}$ \\
\hline *Statistical significance at $\mathrm{P}<0.05$ by multiple regression analysis. & \\
\hline
\end{tabular}

months is required for treatment until a complete recovery of osteomyelitis is achieved in patients with diabetes [10]. Consistent with this, according to Sun et al. [11], patients with Wagner grade 3 ulcers are at risk of LEA. Moreover, Lee et al. [12] also reported that osteomyelitis was a key risk factor associated with LEA. Furthermore, levels of inflammatory markers, such as the WBC count and CRP levels, are also associated with the severity of the wound and the degree of infection, both of which may affect the treatment period. Thus, patients with DF in whom treatment failed had relatively higher WBC counts and CRP levels.

$\mathrm{DN}$ is a common complication of diabetes, and it is also associated with the delayed healing of DF. Patients with renal dysfunction due to DN are vulnerable to impairments in glycolysis in neutrophils. In addition, once accumulated, uremic toxins lead to phagocytic dysfunction. Thus, these patients are vulnerable to impaired infection control [13]. Lewis et al. [14] reported that the progress of chronic kidney disease was an independent risk factor associated with higher mortality due to DF.

In the current study, 7-day mean BG levels had a significant positive correlation with an LHS of $>5$ weeks. Controversy exists regarding whether $\mathrm{HbAlc}$ is a risk factor for developing DF. It has been described as a less important risk factor [8], and has also been considered an important risk factor [15]. Our results showed no significant correlation between $\mathrm{HbAlc}$ levels and an LHS of $>5$ weeks. HbAlc levels are maintained for 6 weeks, and are associated with cardiovascular diseases, retinopathy, neuropathy, and nephropathy [16]. Our results showed that 7-day mean BG levels, rather than $\mathrm{HbAlc}$ levels, had a significant positive correlation with an LHS of $>5$ weeks. It can therefore be inferred that patients who are not easily managed by medical treatment for blood sugar control are vulnerable to prolonged LHS. It has been previously shown that tight glycemic control was effective in reducing the LHS in diabetic patients [17]. 
It has been previously reported that BMI was a risk factor associated with immunologic dysfunction [18]. However, this is not in agreement with our results showing that it had no significant positive correlation with the LHS. In the current study, we enrolled only 22 obese patients (13.4\%) with a mean BMI of $\geq 30$ $\mathrm{kg} / \mathrm{m}^{2}$, while the overall sample of patients with DF had a mean BMI of $25.8 \mathrm{~kg} / \mathrm{m}^{2}$. Smoking is another risk factor for delayed wound healing, which is associated with major vascular disease [19]. However, our results showed no significant correlation between smoking and the LHS. To date, many studies have shown the effects of smoking on delaying wound healing, but these studies have mainly focused on acute wounds. Insufficient data exist regarding the effects of smoking on chronic wounds [19]. According to Steenvoorde et al. [20], smoking did not delay the healing of chronic wounds. Those authors added that a variety of mechanisms are involved in delaying the healing of all types of chronic wounds due to tissue hypoxia, such as vascular insufficiency, arthrosclerosis, and metabolic disturbances. Thus, we could not completely rule out the possibility that the effect of smoking-induced hypoxia might have been masked by poor wound healing conditions in patients with DF.

It is generally believed that longer periods of treatment are needed for wounds in weight-bearing areas, such as the sole, than for those in non-weight-bearing areas. This is because even minor trauma, such as repetitive stress and pressure, may delay wound healing in ulcers [17]. Our results showed, however, that the location of the wound had no significant correlation with the LHS. In patients with DF, even minor injuries due to microvascular or macrovascular disturbances may lead to ulceration in ischemic conditions [21]. It can therefore be inferred that location at a weight-bearing area is not a factor specifically associated with delayed wound healing because it is vulnerable to initial trauma.

Patients with DF are confronted with challenges in resolving foot complications, and are then more vulnerable to a prolonged LHS than non-diabetic patients with similar wounds. Intensive, multidisciplinary, specialized care for such patients would therefore be helpful for shortening the LHS [17]. A multidisciplinary approach is useful in providing the patients with DF with essential elements of DF management; these include wound care, debridement, blood supply, metabolic control, nutritional supplementation, antibiotic therapy, and non-weight-bearing offloading [22]. Moreover, in such an approach, the patients are provided with patient education and assisted self-care with the involvement of family members, which is essential for preventing the occurrence of DF in high-risk patients [23]. Batista et al. [24] suggested that a multidisciplinary approach would be useful for decreasing the morbidity associated with DF. This ex- plains the necessity of multidisciplinary approaches not only for reducing both the LHS and re-admission rate, but also for improving treatment outcomes.

To summarize, our results are as follows: (1) In the Pearson correlation analysis, an LHS of $>5$ weeks had a significant positive correlation with the severity of the wound $(\mathrm{r}=0.647)$, WBC count $(\mathrm{r}=0.571)$, CRP levels $(\mathrm{r}=0.390), \mathrm{DN}(\mathrm{r}=0.020)$, and 7-day mean BG levels $(r=0.120)(P<0.05)$. (2) In the multiple regression analysis, an LHS of $>5$ weeks had a significant positive correlation with the severity of the wound ( $\mathrm{OR}=3.297$; 95\% CI, 1.324-10.483; $\mathrm{P}=0.020)$, WBC count ( $\mathrm{OR}=1.423$; 95\% CI, 0.046-0.356; $\mathrm{P}=0.000)$, CRP levels ( $\mathrm{OR}=1.079 ; 95 \% \mathrm{CI}, 1.015-1.147 ; \mathrm{P}=0.014)$, albumin levels $(\mathrm{OR}=0.263 ; 95 \% \mathrm{CI}, 0.113-3.673 ; \mathrm{P}=0.007)$, and 7 -day mean BG levels (OR $=1.018$; 95\% CI, 1.001-1.035; P = 0.020).

However, our results should be generalized with caution because we enrolled a small series of patients who were hospitalized at a single secondary medical institution. The possibility of selection bias could not therefore be completely ruled out.

In conclusion, our results indicate that surgeons should consider the factors associated with a prolonged LHS in the early management of the patients with DF. Moreover, this should be accompanied by a multidisciplinary approach to reducing the LHS through the effective management of DF by a specialized team of experts from multiple disciplines. To do this, we propose the following: First, diabetologists should play a crucial role in DF care by facilitating tight glycemic control during the treatment of DF. In addition, it is highly probable that patients with DF may exhibit other diabetic complications, such as nephropathy, retinopathy, ischemic heart disease, and cerebrovascular disease. Therefore, these complications should be considered during the choice of an appropriate treatment modality for managing DF. Second, surgeons should initially evaluate and examine patients with DF in order to decide on the clinical pathway and final treatment options. Thus, patients with DF undergo history-taking, a physical examination, biomechanical and laboratory examinations, and imaging studies. Third, patients with DF should receive overall management by experts from other clinical departments. This should also be accompanied by patient education on proper DF care through an intensive nursing program. Fourth, patients with DF should be evaluated for the development of conservative functional or post-reconstructive treatment plans.

\section{CONFLICT OF INTEREST}

No potential conflict of interest relevant to this article was reported. 


\section{REFERENCES}

1. Wild S, Roglic G, Green A, et al. Global prevalence of diabetes: estimates for the year 2000 and projections for 2030 . Diabetes Care 2004;27:1047-53.

2. Jeffcoate WJ, Harding KG. Diabetic foot ulcers. Lancet 2003;361:1545-51.

3. Brem H, Sheehan P, Rosenberg HJ, et al. Evidence-based protocol for diabetic foot ulcers. Plast Reconstr Surg 2006; 117(7 Suppl):193S-209S.

4. Pemayun TGD, Naibaho RM. Clinical profile and outcome of diabetic foot ulcer, a view from tertiary care hospital in Semarang, Indonesia. Diabet Foot Ankle 2017;8:1312974.

5. Ragnarson Tennvall G, Apelqvist J. Health-economic consequences of diabetic foot lesions. Clin Infect Dis 2004;39 Suppl 2:S132-9.

6. Mehta SS, Suzuki S, Glick HA, et al. Determining an episode of care using claims data. Diabetic foot ulcer. Diabetes Care 1999;22:1110-5.

7. Wukich DK, Hobizal KB, Brooks MM. Severity of diabetic foot infection and rate of limb salvage. Foot Ankle Int 2013; 34:351-8,

8. Steed DL, Donohoe D, Webster MW, et al. Effect of extensive debridement and treatment on the healing of diabetic foot ulcers: Diabetic Ulcer Study Group. J Am Coll Surg 1996;183:61-4.

9. Grigoropoulou P, Eleftheriadou I, Jude EB, et al. Diabetic Foot Infections: an Update in Diagnosis and Management. Curr Diab Rep 2017;17:3.

10. Malhotra R, Chan CS, Nather A. Osteomyelitis in the diabetic foot. Diabet Foot Ankle 2014;5.

11. Sun JH, Tsai JS, Huang CH, et al. Risk factors for lower extremity amputation in diabetic foot disease categorized by Wagner classification. Diabetes Res Clin Pract 2012;95:35863.

12. Lee KM, Kim WH, Lee JH, et al. Risk factors of treatment failure in diabetic foot ulcer patients. Arch Plast Surg 2013; 40:123-8.
13. Cheng H, Harris RC. Renal endothelial dysfunction in diabetic nephropathy. Cardiovasc Hematol Disord Drug Targets 2014;14:22-33.

14. Lewis S, Raj D, Guzman NJ. Renal failure: implications of chronic kidney disease in the management of the diabetic foot. Semin Vasc Surg 2012;25:82-8.

15. Margolis DJ, Hofstad O, Feldman HI. Association between renal failure and foot ulcer or lower-extremity amputation in patients with diabetes. Diabetes Care 2008;31:1331-6.

16. Marin-Penalver JJ, Martin-Timon I, Sevillano-Collantes C, et al. Update on the treatment of type 2 diabetes mellitus. World J Diabetes 2016;7:354-95.

17. Puig J, Supervia A, Marquez MA, et al. Diabetes team consultation: impact on length of stay of diabetic patients admitted to a short-stay unit. Diabetes Res Clin Pract 2007;78: 211-6.

18. Winfield RD, Delano MJ, Dixon DJ, et al. Differences in outcome between obese and nonobese patients following severe blunt trauma are not consistent with an early inflammatory genomic response. Crit Care Med 2010;38:51-8.

19. McDaniel JC, Browning KK. Smoking, chronic wound healing, and implications for evidence-based practice. J Wound Ostomy Continence Nurs 2014;41:415-23.

20. Steenvoorde P, Jacobi CE, Van Doorn L, et al. Maggot debridement therapy of infected ulcers: patient and wound factors influencing outcome-a study on 101 patients with 117 wounds. Ann R Coll Surg Engl 2007;89:596-602.

21. Tuttolomondo A, Maida C, Pinto A. Diabetic foot syndrome: immune-inflammatory features as possible cardiovascular markers in diabetes. World J Orthop 2015;6:62-76.

22. American Diabetes Association. Standards of medical care in diabetes: 2011. Diabetes Care 2011;34 Suppl 1:S11-61.

23. Bhattacharya S, Mishra RK. Pressure ulcers: current understanding and newer modalities of treatment. Indian J Plast Surg 2015;48:4-16.

24. Batista F, Augusto Magalhaes A, Gamba M, et al. Ten years of a multidisciplinary diabetic foot team approach in Sao Paulo, Brazil. Diabet Foot Ankle 2010;1:5203. 